\title{
Measurements of Ultracold-Neutron Lifetimes in Solid Deuterium
}

C. L. Morris, ${ }^{1, *}$ J. M. Anaya, ${ }^{1}$ T. J. Bowles, ${ }^{1}$ B.W. Filippone,${ }^{2}$ P. Geltenbort, ${ }^{3}$ R. E. Hill,,${ }^{1}$ M. Hino, ${ }^{4}$ S. Hoedl,${ }^{5}$ G. E. Hogan, ${ }^{1}$ T. M. Ito, ${ }^{2}$ T. Kawai,${ }^{4}$ K. Kirch,,${ }^{1 \dagger}$ S. K. Lamoreaux,${ }^{1}$ C.-Y. Liu, ${ }^{5}$ M. Makela, ${ }^{6}$ L. J. Marek,,${ }^{1}$ J.W. Martin, ${ }^{2}$ R. N. Mortensen, ${ }^{1}$ A. Pichlmaier, ${ }^{1}$ A. Saunders, ${ }^{1}$ S. J. Seestrom, ${ }^{1}$ D. Smith, ${ }^{5,+}$ W. Teasdale, ${ }^{1}$ B. Tipton, ${ }^{2}$ M. Utsuro, ${ }^{4, \S}$ A. R. Young, ${ }^{7}$ and J. Yuan ${ }^{2}$

${ }^{1}$ Los Alamos National Laboratory, Los Alamos, New Mexico 87545

${ }^{2}$ Kellogg Radiation Laboratory, California Institute of Technology, Pasadena, California 91125

${ }^{3}$ Institut Laue-Langevin, BP 156, F-3804 Grenoble Cedex 9, France

${ }^{4}$ University of Kyoto, Kyoto 606-8501, Japan

${ }^{5}$ Princeton University, Princeton, New Jersey 08544

${ }^{6}$ Virginia Polytechnical Institute and State University, Blacksburg, Virginia 24061

${ }^{7}$ North Carolina State University, Raleigh, North Carolina 27695

(Received 26 September 2001; revised manuscript received 3 September 2002; published 18 December 2002)

We present the first measurements of the survival time of ultracold neutrons (UCNs) in solid deuterium $\left(\mathrm{SD}_{2}\right)$. This critical parameter provides a fundamental limitation to the effectiveness of superthermal UCN sources that utilize solid ortho-deuterium as the source material. These measurements are performed utilizing a $\mathrm{SD}_{2}$ source coupled to a spallation source of neutrons, providing a demonstration of UCN production in this geometry and permitting systematic studies of the influence of thermal up-scatter and contamination with para-deuterium on the UCN survival time.

DOI: $10.1103 /$ PhysRevLett.89.272501

PACS numbers: 28.20.Gd, 14.20.Dh, 21.10.Tg, 29.25.Dz

Neutrons with kinetic energies less than $340 \mathrm{neV}$ can be trapped in material bottles and are referred to as ultracold neutrons (UCNs) [1-3]. UCN densities at reactor sources have gradually increased with reactor power and improved techniques for extracting the UCN flux. The highest bottled densities reported in the literature, $41 / \mathrm{cm}^{3}$, have been obtained at the Institut Laue-Langevin (ILL) reactor in Grenoble [4].

Measurements of the neutron electric dipole moment $[5,6]$ and the neutron lifetime [7-9] attest to the utility of bottled UCNs for fundamental experiments with neutrons. UCNs may also prove useful in improved measurements of angular correlations in neutron beta decay $[10,11]$. All of these experimental programs are limited by the available densities of UCNs.

A superthermal UCN source was first proposed in 1975 by Golub and Pendlebury [12] in superfluid ${ }^{4} \mathrm{He}$ and experimentally investigated shortly thereafter $[13,14]$. In this process, phonon creation in the liquid is used to down-scatter cold neutrons to the UCN regime. Upscattering is suppressed by maintaining the superfluid at sufficiently low temperature. Because ${ }^{4} \mathrm{He}$ has no nuclear absorption, the limitations to the density of UCNs accumulated are wall losses and neutron beta decay. The production of UCNs by this process agrees with theoretical expectations [14-18].

While superfluid ${ }^{4} \mathrm{He}$ is an excellent superthermal converter, a few other materials, such as solid deuterium $\left(\mathrm{SD}_{2}\right)$, satisfy the criteria for superthermal production. The limiting UCN density, $\rho_{\mathrm{UCN}}$, one can obtain using a $\mathrm{SD}_{2}$ source is given by the product of the rate of UCN production, $R$, and the lifetime of UCNs in the solid, $\tau_{\mathrm{SD}}$ :
$\rho_{\mathrm{UCN}}=R \tau_{\mathrm{SD}}$. A storage bottle opened to such a source will come into density equilibrium with the density in the solid. This idea led to the concept of a source in which the inside of a neutron bottle is coated with a thin layer of $\mathrm{SD}_{2}$ and the bottle is embedded in a cold-neutron flux $[19,20]$. The volume comes into equilibrium with the UCN density with a time constant for the coupled system, $\tau$, given by

$$
\tau=\tau_{\mathrm{SD}} \frac{V}{V_{\mathrm{SD}}}
$$

where $V_{\mathrm{SD}}$ is the volume of $\mathrm{SD}_{2}$ in the source and $V$ is the total volume of the storage bottle. A valuable summary of $\mathrm{SD}_{2}$ thin-film sources is presented in [21]. The effects of gravity and of the potential of the solid as well as UCN losses other than absorption in the film, neglected in this expression, do not fundamentally alter this picture. The limit to the UCN density is established by the tradeoff between the cold-neutron flux intensity and energy distribution (which determine the production rate) and heating by neutrons and gamma rays in the $\mathrm{SD}_{2}$ and bottle walls, because $\tau_{\mathrm{SD}}$ is a strong function of temperature. However, the predicted production rates in $\mathrm{SD}_{2}$ [20] and lifetimes [22] have not yet been quantitatively verified. Efforts to utilize $\mathrm{SD}_{2}$ sources at reactors identified possible gains but suffered from problems with cooling the solid at full reactor power [13].

Pokotilovski pointed out the advantages of UCN production in $\mathrm{SD}_{2}$ at pulsed neutron sources [23] and showed that UCN densities 2-3 orders of magnitude greater than existing reactor-based UCN sources might be possible. 
The use of spallation as a pulsed source has been suggested $[24,25]$. In a spallation UCN source cold neutrons, produced by moderating spallation neutrons produced in a heavy target by a medium-energy pulsed proton beam, are used to drive a $\mathrm{SD}_{2}$ superthermal converter. The amount of heating for each neutron is lower than in a reactor allowing higher neutron densities in the vicinity of a spallation target to be achieved. Even higher neutron densities can be obtained by pulsing the proton beam and valving off the UCN storage volume from the production volume when the beam is off, using this time to remove heat from the deuterium. The maximum UCN density that is produced is limited by the impulse heating of the $\mathrm{SD}_{2}$. Experiments with the stored UCNs can be performed while the beam is off with low backgrounds.

We have operated a test source with single pulses of protons produced by the LANSCE $800 \mathrm{MeV}$ proton accelerator at Los Alamos National Laboratory. In this Letter, we report the measurements of $\tau_{\mathrm{SD}}$, the lifetime of UCNs in $\mathrm{SD}_{2}$ made using this source. These measurements demonstrate the influences of heating and para-deuterium contamination on the UCN lifetime, and provide a quantitative foundation for the development of $\mathrm{SD}_{2}$ superthermal sources.

A schematic view of our apparatus is shown in Fig. 1. Spallation neutrons were produced in a tungsten target with less than $160 \mathrm{~ns}$ long pulses of $800 \mathrm{MeV}$ protons provided by the LANSCE accelerator. The fast neutron flux was amplified using $(n, 2 n)$ reactions in a beryllium reflector surrounding the spallation target. The spallation neutrons were moderated and cooled in a thin layer of polyethylene surrounding a ${ }^{58} \mathrm{Ni}$-coated stainless steel guide tube with an inner diameter of $7.8 \mathrm{~cm}$. The polyethylene and the bottom of the guide were cooled with

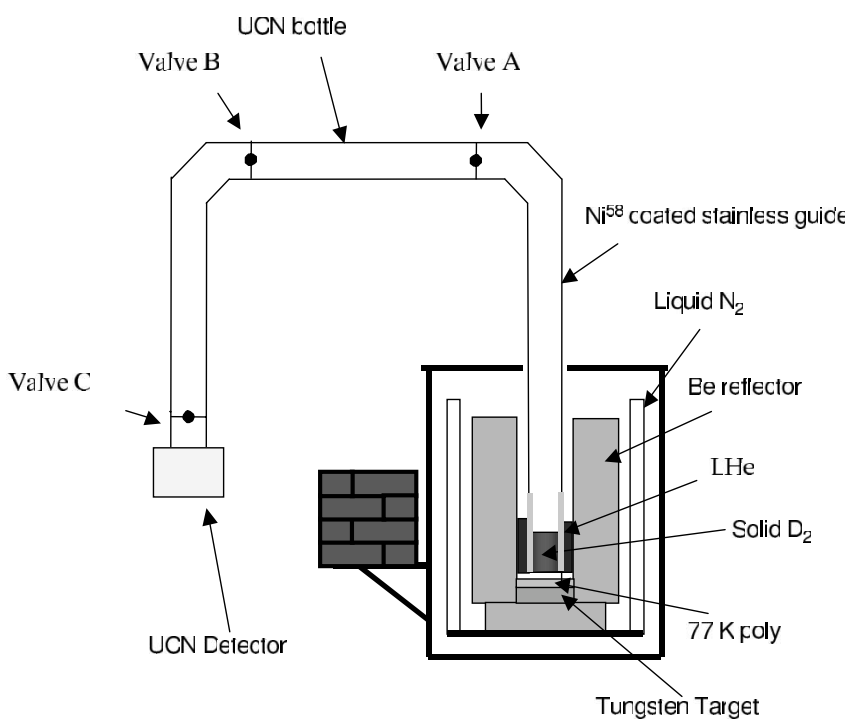

FIG. 1. Schematic view of the apparatus used for this experiment. liquid helium, and a layer of deuterium was frozen on the inside of the guide. UCNs produced in the $\mathrm{SD}_{2}$, confined by the guide tube, could be directed through a series of valves to the UCN detector. Neutrons were detected in a 5-cm-thick multiwire chamber detector filled with a mixture of ${ }^{3} \mathrm{He}$ at 5 mbar and $\mathrm{CF}_{4}$ at 1 bar. The low ${ }^{3} \mathrm{He}$ pressure and the large bend angle in the guide resulted in a high degree of selectivity for detecting UCNs in the apparatus. Data were acquired using a multiscalar that was started by the proton beam passing through a toroidal pickup coil and that scaled the count rate from the detector.

Up-scatter from phonons in the solid [20], up-scatter from para-deuterium molecules in the solid [22], absorption on deuterium, and absorption on hydrogen impurities limit the lifetime of UCNs in $\mathrm{SD}_{2}$. All of these effects have been calculated. The total loss rate is a sum of contributions from these sources:

$$
1 / \tau_{\mathrm{SD}}=1 / \tau_{\text {phonon }}+1 / \tau_{\text {para }}+1 / \tau_{\mathrm{D} \text { abs }}+1 / \tau_{\mathrm{H} \text { abs }},
$$

with the loss rate due to phonon up-scatter having different contributions from the ortho- and para-deuterium in the solid. Establishing the experimental basis to validate these models is essential for the design of a UCN source based on $\mathrm{SD}_{2}$.

$\mathrm{SD}_{2}$ was frozen in the lower part of the cryostat using a helium transfer refrigerator. The temperatures of the lower guide walls and of the liquid-helium cryostat were monitored with an array of silicon diodes. The temperature of the solid was obtained by averaging the temperatures of two diodes mounted on the outside of the guide wall. These tracked the vapor pressure curve of $\mathrm{SD}_{2}$ well at higher temperatures. Later measurements made with diodes embedded in the solid indicate these measurements are accurate to $1 \mathrm{~K}$.

Both the hydrogen contamination and the para-fraction in the $\mathrm{SD}_{2}$ were measured by means of rotational Raman spectroscopy on a gaseous sample taken by warming the deuterium after the measurement [22]. These measurements yielded values for the HD concentrations in the gas that varied from $0.2 \%-0.3 \%$ with an uncertainty of about $0.1 \%$ (all uncertainties quoted at the $67 \%$ confidence level). Other contamination was removed by passing the $\mathrm{D}_{2}$ through a palladium membrane. The para-fraction was controlled by converting the $\mathrm{D}_{2}$ to a near thermal equilibrium ortho/para ratio in an iron-hydroxide-filled cell [26] cooled to a temperature at or slightly below the triple point. In this way, the para-fraction was reduced from a room-temperature equilibrium value of $33 \%$ to $2 \%-4 \%$. Intermediate values were obtained by mixing deuterium at room-temperature equilibrium with converted deuterium before freezing. The precision of the para-fraction measurements was about $1 \%$.

The $\mathrm{SD}_{2}$ volume was measured by integrating the flow of gas while growing the solid. The volume was checked 
when the solid was warmed and the gas was returned to a buffer volume. The overall uncertainty in the volume measurement is approximately $15 \%$.

The sensitivity of the apparatus to UCNs was demonstrated by measuring neutron arrival times with and without a ${ }^{58} \mathrm{Ni}$-coated $0.024-\mathrm{cm}$-thick aluminum foil in place at location $\mathrm{C}$ (in Fig. 1). The ${ }^{58} \mathrm{Ni}$ foil reflects all neutrons with velocities normal to the foil below $8 \mathrm{~m} / \mathrm{s}$. The UCN count rate should drop to near zero with the foil in place. These data are shown in Fig. 2. The number of counts, arriving in a time window between 0.5 and $10 \mathrm{~s}$, with the foil closed was $3 \%$ of the number with the foil opened. About half of these could be attributed to UCN leakage through the gap around the outside of the valve, and the rest were neutrons with normal velocities sufficient to penetrate the potential barrier provided by the ${ }^{58} \mathrm{Ni}$. Thus, the signal in the ${ }^{3} \mathrm{He}$ detector at the end of the guide was predominantly due to UCNs.

If gravity, wall losses, the $\mathrm{SD}_{2}$ potential, and transport effects are neglected, and if the $\mathrm{SD}_{2}$ is thin enough so that its volume is uniformly sampled by the neutrons, the lifetime of neutrons stored in a bottle in contact with $\mathrm{SD}_{2}$ is given by Eq. (1). We have used this idea to measure the UCN lifetime in $\mathrm{SD}_{2}$. As depicted in Fig. 1, valves B and $\mathrm{C}$ were open for these measurements.

Our procedure was to measure the survival probability for UCNs produced and then stored in the "bottle" defined by the end of the guide and valve A, which includes the $\mathrm{SD}_{2}$. First, a specific volume of $\mathrm{SD}_{2}, V_{\mathrm{SD}}$, was frozen into the end of the guide. Data were taken for a range of volumes from 12 to $300 \mathrm{~cm}^{3}$. UCNs were produced and then stored in contact with the $\mathrm{SD}_{2}$ for a time, $t_{s}$. After $t_{s}$, valve A was opened and the surviving UCNs were de-

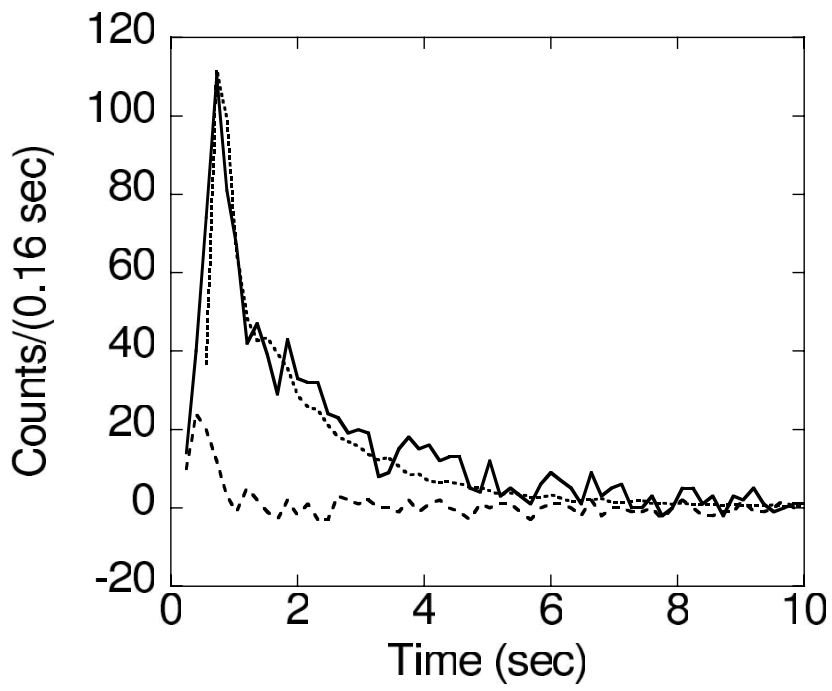

FIG. 2. Background subtracted spectra with (long dashed line) and without (solid line) the ${ }^{58} \mathrm{Ni}$ barrier in the beam at the location of valve $\mathrm{C}$. The short dashed curve is the result of a Monte Carlo calculation of UCN arrival times. tected. From Fig. 2, it is evident that essentially all (greater than 99\%) UCNs arrive in a $10 \mathrm{~s}$ time window after valve $\mathrm{A}$ is opened. Backgrounds were subtracted by fitting a linear function to the data between 20 and $65 \mathrm{~s}$ after the proton trigger and extrapolating "under" the data in the UCN time window. The integral of the background-subtracted counts within the $10 \mathrm{~s}$ window immediately after opening valve A were then taken as the UCN signal for a given $t_{s}$.

For a given $V_{\mathrm{SD}}$, the surviving UCNs were recorded for a series of storage times, $t_{s}=0.5,1.0,2.0$, and $4.0 \mathrm{~s}$. These data were fitted with the form $c_{o} e^{-t / \tau}$, where the parameters $c_{o}$ and $\tau$ were varied to produce the best fit to the data (the $\chi^{2}$ per degree of freedom for the background and the UCN signal fits were all close to 1 and consistent with Poisson statistics). We then systematically varied $V_{\mathrm{SD}}$, the temperature of the $\mathrm{SD}_{2}$, and the ortho/para fraction of the $\mathrm{SD}_{2}$ to produce families of experimentally determined UCN bottle lifetimes, $\tau$.

The UCN lifetimes in the solid deuterium, $\tau_{\mathrm{SD}}$ were extracted from the measured bottle lifetimes, $\tau$, using a Monte Carlo generated lookup table of $\tau_{\mathrm{SD}}$ vs $\tau$. In the Monte Carlo transport, the full experimental geometry, gravitation, the $\mathrm{SD}_{2}$ potential $(108 \mathrm{nV})$, and wall collisions in the guide tube were taken into account. There are a number of parameters that must be determined from the experimental data in order to extract $\tau_{\mathrm{SD}}$ : the probability of UCN loss and nonspecular reflection for each collision with the guide wall, the mean-free path for elastic scattering in the $\mathrm{SD}_{2}, \lambda_{e l}$ (which may depend on the grain size of the sample), and the physical configuration of the $\mathrm{SD}_{2}$ frozen on the walls $\left(\mathrm{SD}_{2}\right.$ can freeze as a flat "pancake" on the bottom of the guide, or coat the walls and bottom of the entire He-cooled surface at the end of the guide, in a "bucket"-shaped configuration). The shape of the $\mathrm{SD}_{2}$ was modeled by a single parameter $r$, which specified the ratio of $\mathrm{SD}_{2}$ volume in a uniform layer coating the walls and bottom and the volume in a pancake at the bottom.

Because samples of $0.4 \mathrm{~cm}$ or less of $\mathrm{SD}_{2}$ were used for most of the lifetime measurements, results were quite insensitive to $\lambda_{e l}$. We therefore used the theoretical value of $\lambda_{e l}=8 \mathrm{~cm}$ for the results presented here.

The ratio of diffuse to specular reflections for wall collisions was adjusted to fit time-of-arrival spectra (see Fig. 2). The remaining transport and physical parameters were adjusted to reproduce the dependence on $V_{\mathrm{SD}}$ for all of the data below $10 \mathrm{~K}$. The volume dependence and timeof-arrival spectra constrained the transport and physical parameters well enough so that the remaining errors in $\tau_{\mathrm{SD}}$ are due to our inability to control systematic uncertainties in the ortho/para ratio and the volume. These have been accounted for by adding an uncertainty of $1 \mathrm{~ms}$ and a relative uncertainty of $20 \%$ in quadrature to all of the extracted deuterium lifetimes. These additions resulted in reduced $\chi^{2}$ of near one in the fitting described below. 


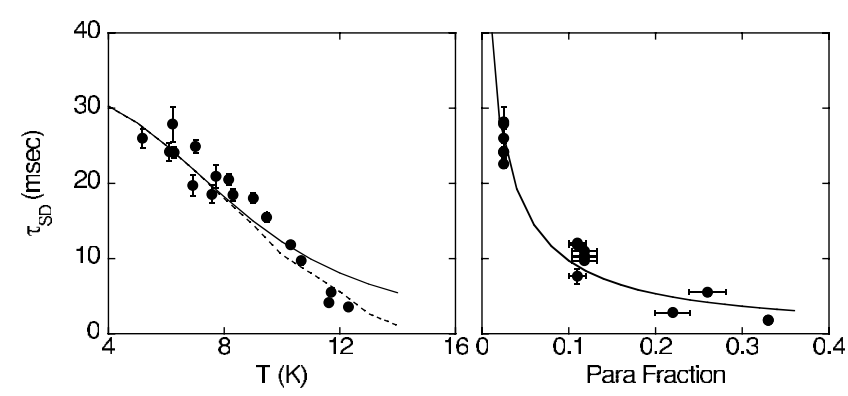

FIG. 3. (left) Data points are measured $\mathrm{SD}_{2}$ lifetimes as a function of temperature, with the para-fraction fixed at $2.5 \%$. Only the statistical errors are shown. Solid lines show the predicted temperature dependence. The dashed line is the predicted effect of departure from the solid lifetime model due to up-scatter from $\mathrm{D}_{2}$ gas in the guide. (right) $\mathrm{SD}_{2}$ lifetimes as a function of para-fraction for all of the data taken below $6 \mathrm{~K}$. The solid line is the model prediction of the para-fraction dependence at an average temperature of $5.6 \mathrm{~K}$.

The lifetimes due to absorption on deuterium and hydrogen were calculated using the known hydrogen contamination, tabulated thermal cross sections, and the $1 / v$ dependence of the cross sections. The lifetimes in solid ortho- and para-deuterium due to temperature upscattering were taken from the literature [20,22]. The lifetime in solid para-deuterium due to molecular transitions was treated as a free parameter and found to be $\tau_{\mathrm{SD}}^{\text {para }}=1.2 \pm 0.14$ (stat) $\pm 0.20($ sys $) \mathrm{ms}$, roughly consistent with a calculation that gives $\tau_{\mathrm{SD}}^{\mathrm{para}}=1.5 \mathrm{~ms}$ [22].

Results for UCN lifetimes $\tau_{\mathrm{SD}}$ in $\mathrm{SD}_{2}$ as a function of the $\mathrm{SD}_{2}$ temperature and para/ortho fractions are shown in Fig. 3. The difference between the solid and the dashed line demonstrates the need to include the effect of deuterium vapor in the guide on the lifetime above $10 \mathrm{~K}$. The measured lifetimes agree well with theoretical predictions of the up-scatter rate. The main contributions to the UCN lifetime in $\mathrm{SD}_{2}$ have been measured and are quantitatively understood. These data demonstrate the potential of a UCN source based on a spallation neutron driven $\mathrm{SD}_{2}$ converter.

We acknowledge the LANSCE operations staff for delivering beam in the new modes needed for this experiment and Warren Pierce for his excellent support. We also acknowledge fruitful conversations with R. Golub and with A. Serebrov. This work has been supported by the DOE LDRD program and by the NSF 9420470, 9600202, 9807133, 0071856.

\footnotetext{
*Electronic address: CMorris@lanl.gov

†Present address: Paul Scherrer Institut, Villigen, Switzerland.

${ }^{\ddagger}$ Present address: Stanford Linear Accelerator Center, Menlo Park, California.
}

${ }^{\S}$ Present address: Research Center for Nuclear Physics, Osaka University, Osaka, Japan.

[1] Y. B. Zel'dovich, Sov. Phys. JETP 9, 1389 (1959).

[2] V. I. Lushchikov, Yu. N. Pokotilovsky, A. V. Strelkov, and F. L. Shapiro, JETP Lett. 9, 23 (1969).

[3] A. Steyerl, Phys. Lett. 29B, 33 (1969).

[4] A. Steyerl et al., Phys. Lett. A 116, 347 (1986).

[5] P. G. Harris et al., Phys. Rev. Lett. 82, 904 (1999).

[6] I. S. Altarev, Y.V. Borisov, N. V. Borovikova, A. I. Egorov, S. N. Ivanov, E. A. Kolomensky, M. S. Lasakov, V. M. Lobashev, and V. A. Nazarenko, Phys. At. Nucl. 59, 1152 (1996).

[7] W. Mampe, L. N. Bondarenko, V. I. Morozov, Y. N. Panin, and A. I. Fomin, JETP Lett. 57, 82 (1993).

[8] S. Arzumanov, L. Bondarenko, S. Chernyavsky, W. Drexel, A. Fomin, P. Geltenbort, V. Morozov, Y. Panin, J. Pendlebury, and K. Schreckenbach, Phys. Lett. B 483, 15 (2000).

[9] A. Pichlmaier, J. Butterworth, P. Geltenbort, H. Nagel, V. Nesvizhevsky, S. Neumaier, K. Schreckenbach, and E. Steichele, Nucl. Instrum. Methods Phys. Res., Sect. A 440, 517 (2000).

[10] T. Bowles and A. R. Young, co-Principal Investigators, A proposal for an accurate measurement of the neutron spin-electron angular correlation in polarized neutron beta decay with ultracold neutrons (2000).

[11] S. Gardner and C. Zhang, Phys. Rev. Lett. 86, 5666 (2001).

[12] R. Golub and J. M. Pendlebury, Phys. Lett. 53A, 133 (1975).

[13] I. S. Altarev et al., Phys. Lett. 80A, 413 (1980).

[14] R. Golub, C. Jewell, P. Ageron, W. Mampe, B. Heckel, and I. Kilvington, Z. Phys. B 51, 187 (1983).

[15] H. Yoshiki, K. Sakai, M. Ogura, T. Kawai, Y. Masuda, T. Nakajima, T. Takayama, S. Tanaka, and A. Yamaguchi, Phys. Rev. Lett. 68, 1323 (1992).

[16] A. I. Kilvington, R. Golub, W. Mampe, and P. Ageron, Phys. Lett. A 125, 416 (1987).

[17] P. R. Huffman, C. R. Brome, J.S. Butterworth, K. J. Coakley, M. S. Dewey, S. N. Dzhosyuk, R. Golub, G. L. Greene, K. Habicht, and S. K. Lamoreaux, Nature (London) 403, 62 (2000).

[18] C. R. Brome et al., Phys. Rev. C 63, 055502 (2001).

[19] R. Golub and K. Böning, Z. Phys. B 51, 95 (1983).

[20] Z.-C. Yu, S. S. Malik, and R. Golub, Z. Phys. B 62, 137 (1986).

[21] R. Golub, D. J. Richardson, and S. K. Lamoreaux, UltraCold Neutrons (Hilger, Bistol, 1991), p. 62.

[22] C.-Y. Liu, A. R. Young, and S. K. Lamoreaux, Phys. Rev. B 62, R3581 (2000).

[23] Y. N. Pokotilovski, Nucl. Instrum. Methods Phys. Res., Sect. A 356, 412 (1995).

[24] A. P. Serebrov, V. A. Mityukhlyaev, A. A. Zakharov, T. Bowles, G. Greene, and J. Sromicki, JETP Lett. 66, 802 (1997).

[25] A. P. Serebrov, V. Mityukhlyaev, A. Zakharov, A. Kharitonov, V. Shustov, V. Kuzminov, M. Lasakov, R. Taldaev, A. Aldushchenkov, and V. Varlamov, Nucl. Instrum. Methods Phys. Res., Sect. A 440, 658 (2000).

[26] D. H. Weitzel and O. E. Park, Rev. Sci. Instrum. 27, 56 (1956). 